\title{
An SPH model for free surface flows with moving rigid objects
}

\author{
M. B. $\operatorname{Liu}^{1, *, \dagger}$ J. R. Shao ${ }^{1}$ and H. Q. Li $^{2}$ \\ ${ }^{1}$ Key Laboratory for Mechanics in Fluid-Solid Coupling Systems, Institute of Mechanics, Chinese Academy of Sciences, \\ Beijing 100190, China \\ ${ }^{2}$ School of Information and Electronics, Beijing Institute of Technology, Beijing 100081, China
}

\begin{abstract}
SUMMARY
This paper presents a computational model for free surface flows interacting with moving rigid bodies. The model is based on the SPH method, which is a popular meshfree, Lagrangian particle method and can naturally treat large flow deformation and moving features without any interface/surface capture or tracking algorithm. Fluid particles are used to model the free surface flows which are governed by Navier-Stokes equations, and solid particles are used to model the dynamic movement (translation and rotation) of moving rigid objects. The interaction of the neighboring fluid and solid particles renders the fluid-solid interaction and the non-slip solid boundary conditions. The SPH method is improved with corrections on the SPH kernel and kernel gradients, enhancement of solid boundary condition, and implementation of Reynoldsaveraged Navier-Stokes turbulence model. Three numerical examples including the water exit of a cylinder, the sinking of a submerged cylinder and the complicated motion of an elliptical cylinder near free surface are provided. The obtained numerical results show good agreement with results from other sources and clearly demonstrate the effectiveness of the presented meshfree particle model in modeling free surface flows with moving objects. Copyright @ 2013 John Wiley \& Sons, Ltd.
\end{abstract}

Received 14 June 2013; Revised 28 October 2013; Accepted 5 November 2013

KEY WORDS: smoothed particle hydrodynamics (SPH); free surface flow, meshfree particle method; moving rigid objects; fluid-solid interaction

\section{INTRODUCTION}

Free surface flows with moving rigid objects can be frequently observed in daily life, engineering and sciences and can be of utmost importance both in theory and practice. Typical examples include landing of aircrafts onto water, diving of sportsman, movement of ships and floating platforms on the sea and sloshing of liquid containers due to external excitations. Numerical simulation of free surface flows with moving objects involves rapid movement of solid objects, changing and breakup of free surfaces, strong turbulence and vortices and violent fluid-solid interaction. Conventional Lagrangian grid-based methods such as the FEM is difficult in treating large flow deformations, while conventional Eulerian grid-based methods such as the finite volume method and finite difference method need special algorithms to track or capture the moving free surfaces and moving objects, which usually complicate the problem [1-4].

SPH is usually regarded as the oldest modern meshfree particle method originally used for continuum scale applications. As a 'truly' meshfree, particle method, it does not need any mesh or grid either for generating initial data or for transferring information from the background mesh to particles. SPH was first invented to solve astrophysical problems in three-dimensional open space $[5,6]$ and later extended for many other problems. In SPH, the state of a system is represented by a set of

\footnotetext{
*Correspondence to: M. B. Liu, Key Laboratory for Mechanics in Fluid-Solid Coupling Systems, Institute of Mechanics, Chinese Academy of Sciences, Beijing 100190, China.

†E-mail: liumoubin@imech.ac.cn 
particles, which possess material properties and interact with each other within a range controlled by a weight function or smoothing function $[7,8]$. As a Lagrangian particle method, SPH conserves mass exactly. In SPH, there is no explicit interface tracking for multiphase flows-the motion of the fluid is represented by the motion of the particles, and fluid surfaces or fluid-fluid interfaces move with particles representing their phase defined at the initial stage. The meshfree nature of SPH method removes the difficulties due to large deformations because SPH uses particles rather than mesh as a computational frame to approximate-related governing equations.

There are a few literatures addressing the application of SPH method to free surface flows interacting with moving rigid bodies. For example, Oger et al. studied the entry of water of a free-falling wedge using a weakly compressible SPH (WCSPH) model [9], while Shao numerically simulated similar water entry problems using an incompressible SPH (ISPH) model [10]. Iglesias et al. simulated the anti-roll tanks and sloshing type problems [11]. Rhee and Engineer studied liquid tank sloshing with Reynolds-averaged Navier-Stokes (RANS) [12]. Marrone et al. even presented a $2 \mathrm{D}+t \mathrm{SPH}$ model to study the breaking wave pattern generated by fast-moving ships [13].

In this paper, an improved SPH model for numerical simulation of free surface flows interacting with moving rigid bodies shall be presented. The SPH model includes an improved approximation scheme with corrections on kernel gradient and density, an enhanced solid boundary treatment (SBT) algorithm, and the RANS turbulence model. The effectiveness of the SPH model shall be carefully validated by three typical examples, including the water exit of a cylinder, the sinking of a submerged cylinder and the complicated motion of an elliptical cylinder near free surface.

\section{GOVERNING EQUATIONS}

\subsection{Lagrangian form of Reynolds-averaged Navier-Stokes equations for viscous flows}

In this paper, the Lagrangian form of the RANS equations is used as governing equations for incompressible, viscous flows $[14,15]$.

$$
\begin{gathered}
\frac{d \rho}{d t}=-\rho \nabla \cdot \boldsymbol{v}, \\
\frac{d v}{d t}=-\frac{1}{\rho} \nabla P+\frac{\mu}{\rho} \nabla^{2} \boldsymbol{v}+\boldsymbol{g}+\frac{1}{\rho} \nabla(\rho \boldsymbol{R}),
\end{gathered}
$$

where $t$ is time, $\rho$ is fluid density, $v$ is the velocity vector, $P$ is pressure, $\mu$ is the dynamic viscosity, $\boldsymbol{g}$ is the gravitational acceleration and $\boldsymbol{R}$ is the Reynolds stress tensor whose elements are $R_{i j}=-\overline{v_{i}^{\prime} v_{j}^{\prime}}$. The eddy viscosity assumption $[14,15]$ is used to model the Reynolds stress tensor as

$$
\boldsymbol{R}=2 v_{t} \boldsymbol{S}-\frac{2}{3} k \boldsymbol{I}
$$

where $v_{t}$ is the eddy viscosity, $S$ is the mean rate-of-strain tensor, $k$ is turbulence kinetic energy and $\boldsymbol{I}$ is a unit tensor. The Smagorinsky model of eddy viscosity $v_{t}=\left(C_{s} \Delta l\right)^{2} \sqrt{2 S_{i j} S_{i j}}$ is widely used, where $C_{s}$ is the Smagorinsky constant usually taken as 0.12 and $\Delta l$ is a mixing length which is assumed to be the initial particle spacing in SPH. $S_{i j}$ are the elements of $\boldsymbol{S}$ given by

$$
S_{i j}=\frac{1}{2}\left(\frac{\partial v_{j}}{\partial x_{i}}+\frac{\partial v_{i}}{\partial x_{j}}\right)
$$

Considering $k=\overline{v_{i}^{\prime} v_{j}^{\prime}} / 2$ and $R_{i j}=-\overline{v_{i}^{\prime} v_{j}^{\prime}}$, the relation between $k$ and the Reynolds stress tensor can be written as $k=-R_{i i} / 2$. 


\subsection{Equation of motion for moving rigid body}

For a moving rigid body, the equation of motion is simply the Newton's law of motion. The center of mass can be written as follows:

$$
\frac{d \boldsymbol{u}_{o}}{d t}=\frac{\boldsymbol{F}}{M}+\boldsymbol{g},
$$

where $\boldsymbol{u}_{o}$ is the velocity of the center of mass, $\boldsymbol{F}$ is the summation of forces without gravity around the rigid body and $M$ is the mass of the rigid body. For two-dimensional problems, the equation for the angular velocity is

$$
\frac{d \omega}{d t}=\frac{J}{I}
$$

where $\omega$ is the angular velocity, $I$ is the moment of inertia and $\boldsymbol{J}$ is the total moment of force on the rigid body about the center of mass. The velocity of a point $a$ on the rigid body is

$$
\boldsymbol{u}_{a}=\boldsymbol{u}_{o}+\omega \times \boldsymbol{r}_{o a},
$$

where $\boldsymbol{r}_{o a}$ is the vector from the center of mass $o$ to point $a$.

\section{SPH METHODOLOGY}

\subsection{SPH equations of motion}

SPH method uses a set of particles to represent the state of a simulated system. The particles possess individual material properties, move according to corresponding governing conservation equations and act as the computational frame for field variable approximations. Therefore, the SPH approximation does not require a predefined mesh to provide any connective of the particles in the process of computation. The SPH method consists two steps in obtaining an SPH formulation, kernel and particle approximations. The kernel approximation is to represent a function and its derivatives in continuous form as integral representation using the smoothing function and its derivatives. In the particle approximation, the computational domain is discretized with a set of particles. A field function and its derivative can then be written in the following forms. More detailed descriptions of the SPH method can be found in $[16,17]$.

Specifically, in SPH, a field function and its derivative can then be written in the following forms:

$$
\begin{gathered}
<f\left(\boldsymbol{x}_{i}\right)>=\sum_{j=1}^{N} \frac{m_{j}}{\rho_{j}} f\left(\boldsymbol{x}_{j}\right) W\left(\boldsymbol{x}_{i}-\boldsymbol{x}_{j}, h\right), \\
<\nabla f\left(\boldsymbol{x}_{i}\right)>=\sum_{j=1}^{N} \frac{m_{j}}{\rho_{j}} f\left(\boldsymbol{x}_{j}\right) \nabla_{i} W_{i j},
\end{gathered}
$$

where $<f\left(\boldsymbol{x}_{i}\right)>$ is the approximated value of particle $i, f\left(\boldsymbol{x}_{j}\right)$ is the value of $f(\boldsymbol{x})$ associated with particle $j, \boldsymbol{x}_{i}$ and $\boldsymbol{x}_{j}$ are the positions of corresponding particles, $m$ denotes mass, $h$ is the smooth length, $N$ is the number of the particles in the support domain and $W$ is the smoothing function representing a weighted contribution of particle $j$ to particle $i$. In this paper, the frequently used cubic spline is used [8].

Substituting the SPH approximations for a function and its derivative to the RANS equations, after some trivial transformation, the SPH equations of motion for viscous fluid flows can be obtained as

$$
\frac{d \rho_{i}}{d t}=\sum_{j=1}^{N} m_{j} \boldsymbol{v}_{i j} \nabla_{i} W_{i j},
$$




$$
\begin{aligned}
\frac{d \boldsymbol{v}_{i}}{d t}= & -\sum_{j=1}^{N} m_{j}\left(\frac{p_{i}}{\rho_{i}^{2}}+\frac{p_{j}}{\rho_{j}^{2}}\right) \nabla_{i} W_{i j}+\sum_{j=1}^{N} \frac{4 m_{j}\left(\mu_{i}+\mu_{j}\right) \boldsymbol{x}_{i j} \bullet \nabla_{i} W_{i j}}{\left(\rho_{i}+\rho_{j}\right)^{2}\left(x_{i j}^{2}+0.01 h^{2}\right)} v_{i j} . \\
& +\sum_{j} m_{j}\left(\frac{\boldsymbol{R}_{i}}{\rho_{i}}+\frac{\boldsymbol{R}_{j}}{\rho_{j}}\right) \nabla_{i} W_{i j}+\boldsymbol{g}
\end{aligned}
$$

where the first, second and third terms on the left-hand side of equation (11) describe the SPH approximations for pressure, viscosity and Reynolds stress tensor, respectively.

For the motion of moving rigid body, $\boldsymbol{F}$ and $\boldsymbol{J}$ can be expressed in SPH as follows:

$$
\begin{gathered}
\boldsymbol{F}=\sum_{b} \boldsymbol{f}_{b}, \\
\boldsymbol{J}=\sum_{b} \boldsymbol{r}_{o b} \times \boldsymbol{f}_{b},
\end{gathered}
$$

where $f_{b}$ denotes the force on particle $b$ because of fluids. The summation is over all rigid body particles.

\subsection{Modeling incompressible flow in $\mathrm{SPH}$}

There are basically two approaches in modeling incompressible fluid flows in SPH. The most frequently used approach is based on the assumption that the modeling fluid can be regarded as weakly compressible, and this approach is usually referred to as WCSPH [18]. The particle density is slightly changed while the particle pressure can be calculated from an artificial equation of state. WCSPH is simple in concept, easy in computer implementation, and it is able to obtain reasonable flow patterns, but it encounters pressure oscillations. This leads to the development of another approach to model the free surface incompressible flows as rigorously incompressible rather than weakly compressible as in WCSPH [19]. This leads to the projection-based ISPH method [20,21]. Unlike WCSPH, the particle density in ISPH remains unchanged, ensuring the incompressibility of the modeling fluid. The pressure is implicitly obtained from solving the pressure Poisson's equation (PPE) rather than from an artificial equation of state as in WCSPH. The PPE is only related on the relative positions and relative velocities between particles without artificial parameters. Meanwhile, the solution of PPE in ISPH is based on the entire computational domain. Therefore, compared with traditional WCSPH methods, the ISPH methods are usually able to obtain much smoother pressure fields [22]. The resulting equations in ISPH are generally cumbersome, and the computational cost is usually tremendous. What is more, with the exact implementation of the constraint of constant density, the entire particle system in ISPH is not as flexible as that in WCSPH. One notable example is that in ISPH, the free surface condition needs to be explicitly and rigorously implemented.

During recent years, great progresses have been made in WCSPH methods, and the previous defections such as pressure oscillations have been resolved quite well either by using high-order SPH approximation schemes such as the corrective smoothed particle method by Chen et al. [23] and the finite particle method by Liu et al. $[24,25]$ or by using a well-tuned artificial viscosity [26]. Density re-initialization $[27,28]$ is another effective approach to reduce large oscillation of the particle pressure and can be applied every several time steps without too much computational cost. Furthermore, the improvements in treating solid boundaries [29] and free surfaces [30] also contribute to WCSPH in better modeling free surface incompressible flows. Therefore, in this work, we choose to use WCSPH for modeling the free surface incompressible flows with moving object.

The artificial compressibility considers that every theoretically incompressible fluid is actually compressible. Therefore, it is feasible to use a quasi-incompressible equation of state to model the incompressible flow. The purpose of introducing the artificial compressibility is to produce the time derivative of pressure. In this work, the artificial equation of state is

$$
p=c^{2} \rho,
$$


where $c$ is the sound speed, which is a key factor that deserves careful consideration. If the actual sound speed is employed, the real fluid is approximated as an artificial fluid, which is ideally incompressible. Monaghan [31] argued that the relative density variation $\delta$ is related to the fluid bulk velocity and sound speed in the following way:

$$
\delta=\frac{\Delta \rho}{\rho_{0}}=\frac{\left|\rho-\rho_{0}\right|}{\rho_{0}}=\frac{V_{b}^{2}}{c^{2}}=M^{2},
$$

where $\rho_{0}, \Delta \rho, V_{b}$ and $M$ are the initial density, absolute density variation, fluid bulk velocity and Mach number, respectively.

Morris et al. [32], through considering the balance of pressure, viscous force and body force, proposed an estimate for the sound speed. He argued that the square of the sound speed should be comparable with the largest value of $V_{b}^{2} / \delta, v V_{b} / \delta l$ and $F l / \delta$, that is,

$$
c^{2}=\max \left(\frac{V_{b}^{2}}{\delta}, \frac{v V_{b}}{\delta l}, \frac{F l}{\delta}\right),
$$

where $v(v=\mu / \rho)$ is the kinetic viscosity, $F$ is the magnitude of the external body force and $l$ is the characteristic length scale.

\subsection{Density and kernel gradient correction}

Free surface flows interacting with moving rigid body are usually associated with changing and breakup of free surfaces. When wave front violently impacts onto solid walls or moving rigid bodies, water particles can first be splashed away from bulky fluid and then fall onto the bulky fluid. The changing and breakup of free surfaces as well as splashing and fall of water particles lead to highly disordered particle distribution, which can seriously influence computational accuracy of SPH approximations. Hence, an SPH approximation scheme, which is of higher order accuracy and is insensitive to disordered particle distribution, is necessary.

In this work, we used two modified schemes for approximating density (density correction, or DC) and kernel gradient (kernel gradient correction, or KGC). As to the density correction, a re-normalization approach is used, in which the density can be approximated as

$$
\begin{gathered}
\rho_{i}^{\text {new }}=\sum_{j=1}^{N} \rho_{j} W_{i j}^{\text {new }} \frac{m_{j}}{\rho_{j}}=\sum_{j=1}^{N} m_{j} W_{i j}^{\text {new }}, \\
W_{i j}^{\text {new }}=\frac{W_{i j}}{\sum_{j=1}^{N} W_{i j} \frac{m_{j}}{\rho_{j}}} .
\end{gathered}
$$

In the KGC technique, a modified or corrected kernel gradient is obtained by multiplying the original kernel gradient with a local reversible matrix $\boldsymbol{L}\left(\boldsymbol{r}_{i}\right)$, which is obtained from Taylor series expansion method. In two-dimensional spaces, the new kernel gradient of the smoothing function $\nabla_{i}^{C} W_{i j}$ can be obtained as follows:

$$
\begin{gathered}
\nabla_{i}^{C} W_{i j}=L\left(\boldsymbol{r}_{i}\right) \nabla_{i} W_{i j}, \\
L\left(\boldsymbol{r}_{i}\right)=\left(\sum_{j}\left(\begin{array}{ll}
x_{j i} \frac{\partial W_{i j}}{\partial x_{i}} & y_{j i} \frac{\partial W_{i j}}{\partial x_{i}} \\
x_{j i} \frac{\partial W_{i j}}{\partial y_{i}} & y_{j i} \frac{\partial W_{i j}}{\partial y_{i}}
\end{array}\right) V_{j}\right)^{-1},
\end{gathered}
$$

where $x_{j i}=x_{j}-x_{i}, y_{j i}=y_{j}-y_{i}$. It is found that for general cases with irregular particle distribution, variable smoothing length, and truncated boundary areas, the SPH particle approximation scheme with KGC is of second-order accuracy [33]. 


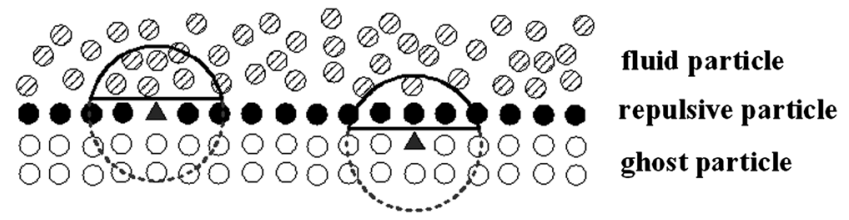

Figure 1. Illustration of the coupled dynamic solid boundary treatment algorithm.

\subsection{Solid boundary treatment}

In this work, we used a coupled dynamic-SBT (CD-SBT) algorithm [29], in which two types of virtual particles, repulsive particles and ghost particles (as shown in Figure 1), are used to represent the solid boundary. The repulsive particles produce a suitable repulsive force to the approaching fluid particles near the boundary, and they are located right on the solid boundary. Ghost particles are located outside the solid boundary area. Different from conventional SBT algorithms, in which ghost particles are generated by mirroring or reflecting fluid particles onto solid boundary areas, and need to adapt with the fluid particles at each time step, this new SBT algorithm can generate ghost particles in a regular or irregular distribution at the first time step, while ghost particle positions do not need to change during following steps.

The CD-SBT algorithm consists of a new repulsive force for repulsive particles and a new numerical scheme to approximate the information of the virtual particles. The new repulsive force is a distance-dependent repulsive force with finite magnitude on fluid particles approaching solid boundaries. It is an improvement on Kourosh's work [34], which needs to calculate the tangential and normal directions of solid boundaries, and is not easy to implement for complex geometries. The improved repulsive force is a finite distance-dependent repulsive force on fluid particles approaching solid boundaries. To restore the consistency of SPH particle approximation, KGC, Shepard filter method or moving least square method can be used in the CD-SBT algorithm for approximating both the fluid and virtual particles. More detailed descriptions can be found in [29].

\subsection{Fluid-solid interface treatment}

Though the SPH method has been widely applied to different problems in engineering and sciences, there are few works discussing fluid-structure interactions with SPH method. Fluid-solid interface is usually treated using some kind of interface force. The interface force is usually dependent on the interface curvature, and it needs to calculate the tangential and normal directions. This is usually difficult to approximate in SPH.

In this work, we have further extended the CD-SBT algorithm to the treatment of fluid-solid interface and refer to the algorithm as coupled dynamic fluid-solid interface treatment (CD-FSIT). It is also necessary to distribute ghost particles on the rigid body, accumulate force and momentum on the ghost particles and accumulate them on the rigid body to drive its motion. The only difference is that in CD-SBT, the solid particles are fixed, while in CD-FSIT, the solid particles move with the rigid objects. The CD-FSIT algorithm also includes a soft repulsive force between fluid and solid particles and higher order particle approximate scheme to obtain the information of the virtual particles. As such, the implementation of fluid-structure interaction in this work is straightforward and can lead to smooth and accurate pressure field in the flow domain and pressure loads on the moving objects. More detailed descriptions on the CD-FSIT can be found in [35].

\section{NUMERICAL EXAMPLES}

\subsection{Water exit of a cylinder}

In this section, water exit of a cylinder will be numerically simulated using the presented SPH model. Many researchers have conducted similar studies on water exit using different approaches. For example, Greenhow and Moyo provided detailed investigations on water entry and exit of horizontal 
circular cylinders [4] by using a two-dimensional BEM based on irrotational flow assumption. Lin also presented a numerical simulation of water exit of a cylinder using a cut-cell technique with a fixed grid [1]. In Lin's work, the movement of rigid body is tracked by Lagrangian method, and the fluid motion around the body is solved by Eulerian method. Also, a rigid body is approximated by the partial cell treatment, which treats the irregular body by using the volumetric fraction of solid in Cartesian cells. Tyvand and Miloh provided theoretical results for this water exit problem [36].

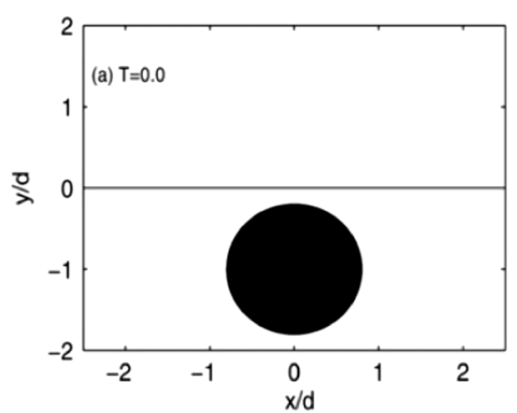

(a)
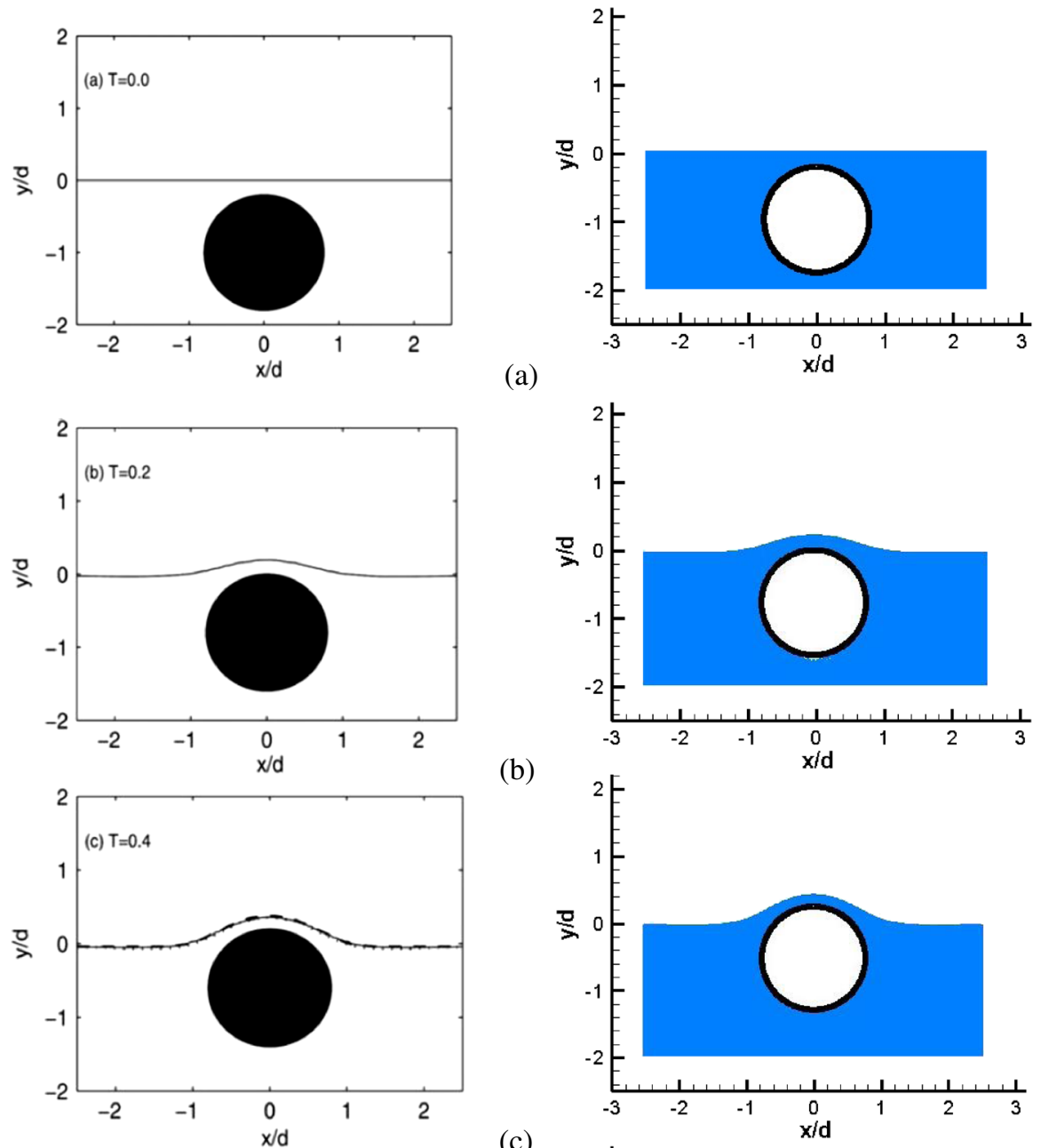

(b)
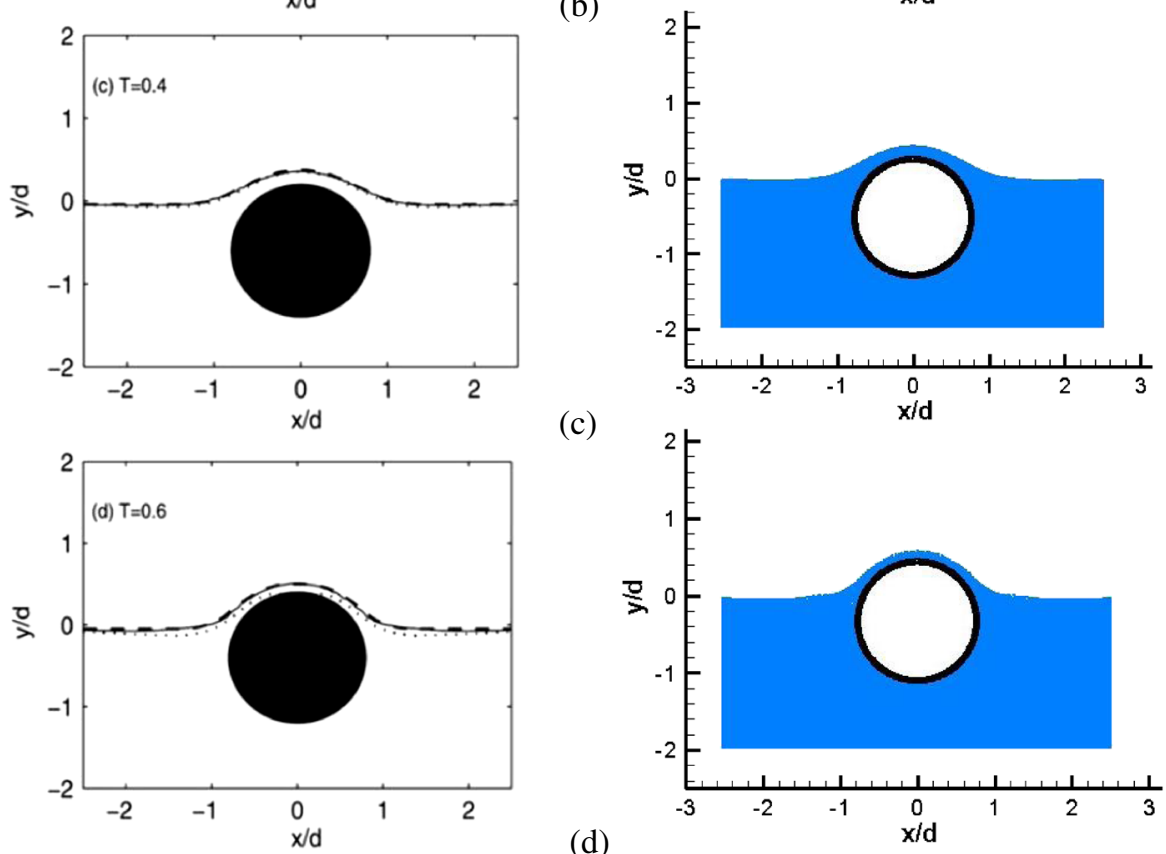

(c)

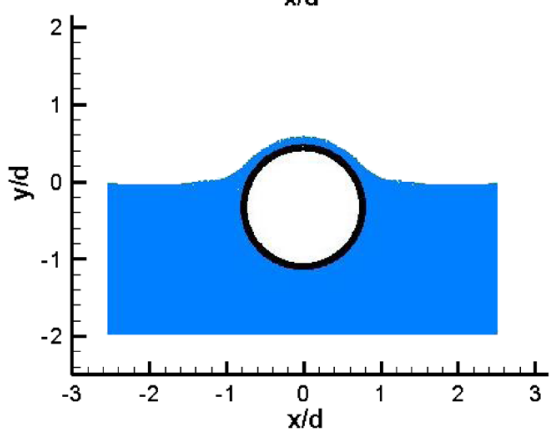

Figure 2. Simulation results of the water exit of a horizontal cylinder with comparisons among results from other sources at different time instants $(\mathrm{a}: \mathrm{T}=0.0 ; \mathrm{b}: \mathrm{T}=0.2 ; \mathrm{c}: \mathrm{T}=0.4 ; \mathrm{d}: \mathrm{T}=0.6 ; \mathrm{e}: \mathrm{T}=0.8 ; \mathrm{f}: \mathrm{T}=1.0$; $\mathrm{g}: \mathrm{T}=2.0$ and $\mathrm{h}: \mathrm{T}=3.0)$. Left: numerical results by Lin (solid line), numerical results by Greenhow and Moyo (dashed line) and theoretical results by Tyvand and Miloh (dotted line) (from [1]). Right: present SPH results. 


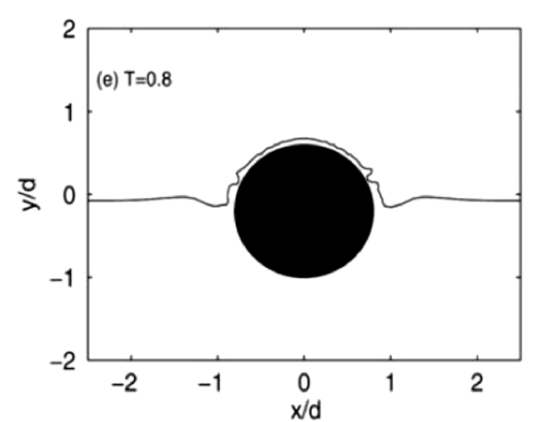

(e)
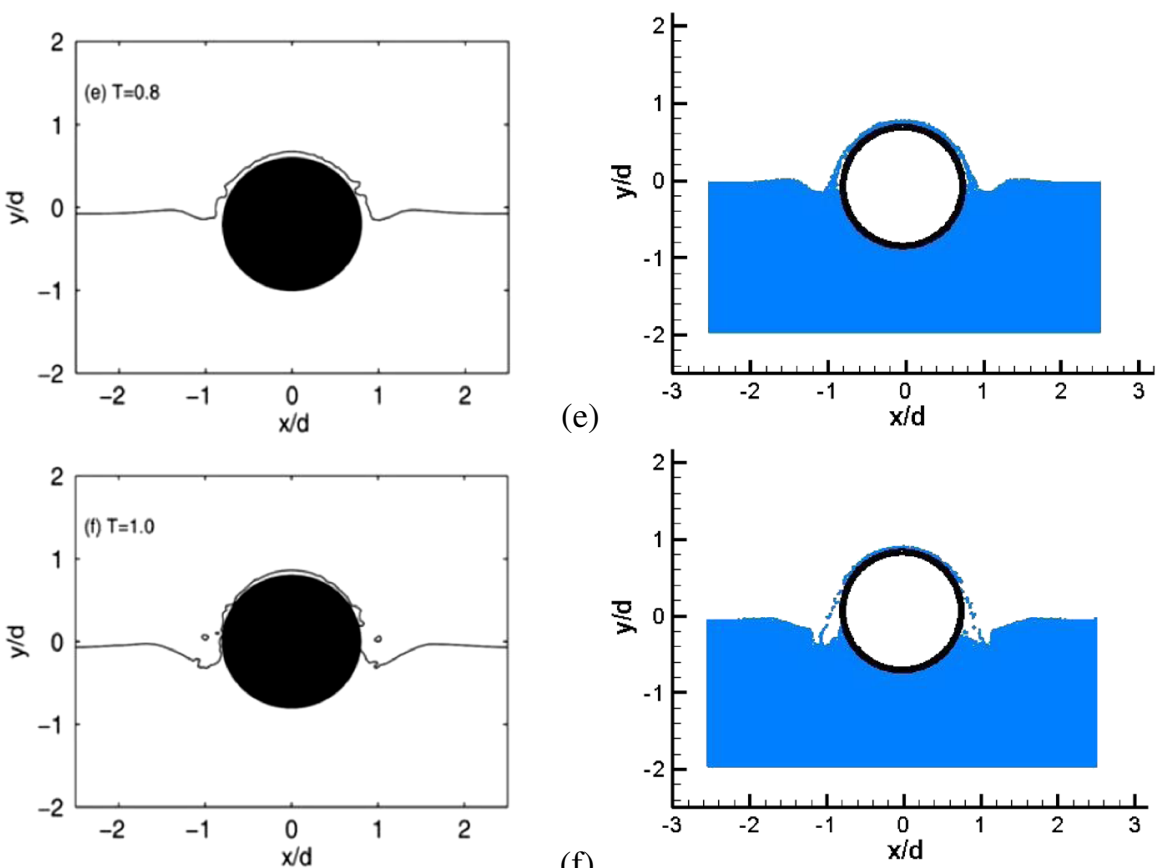

(f)
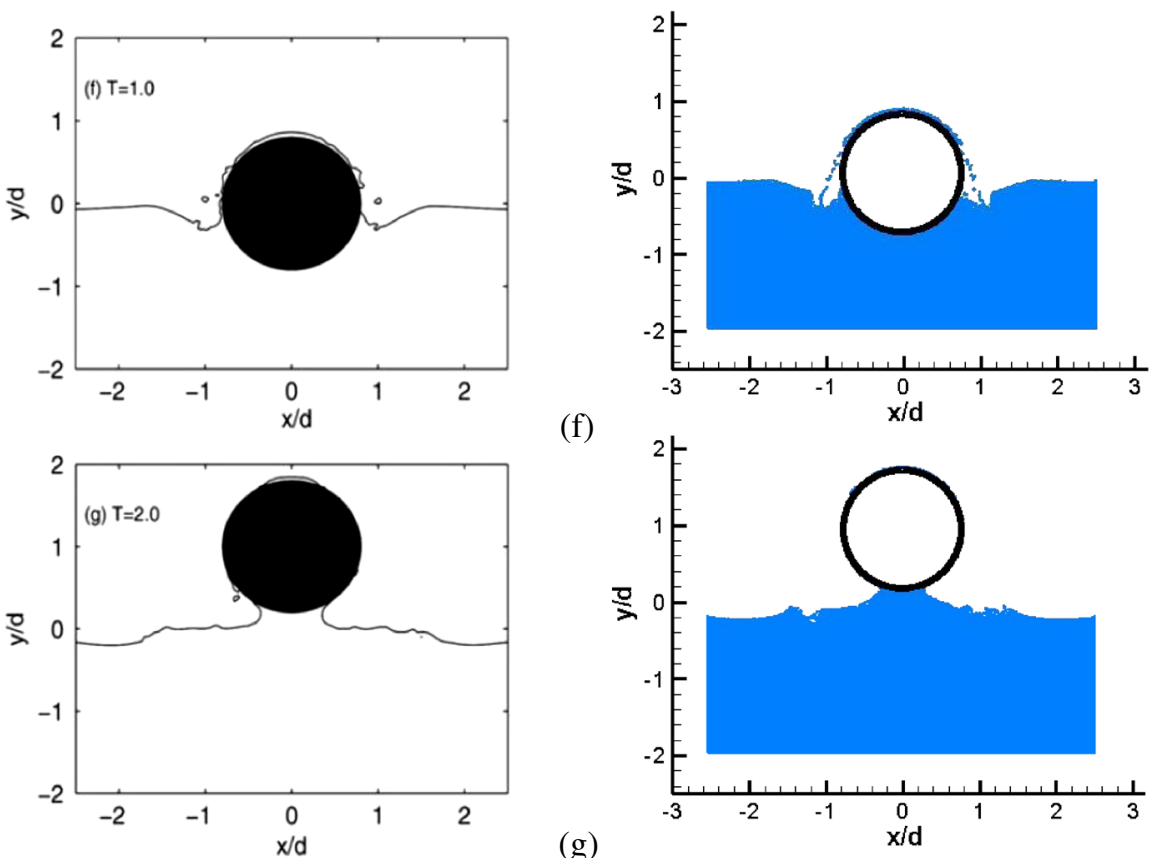

(g)
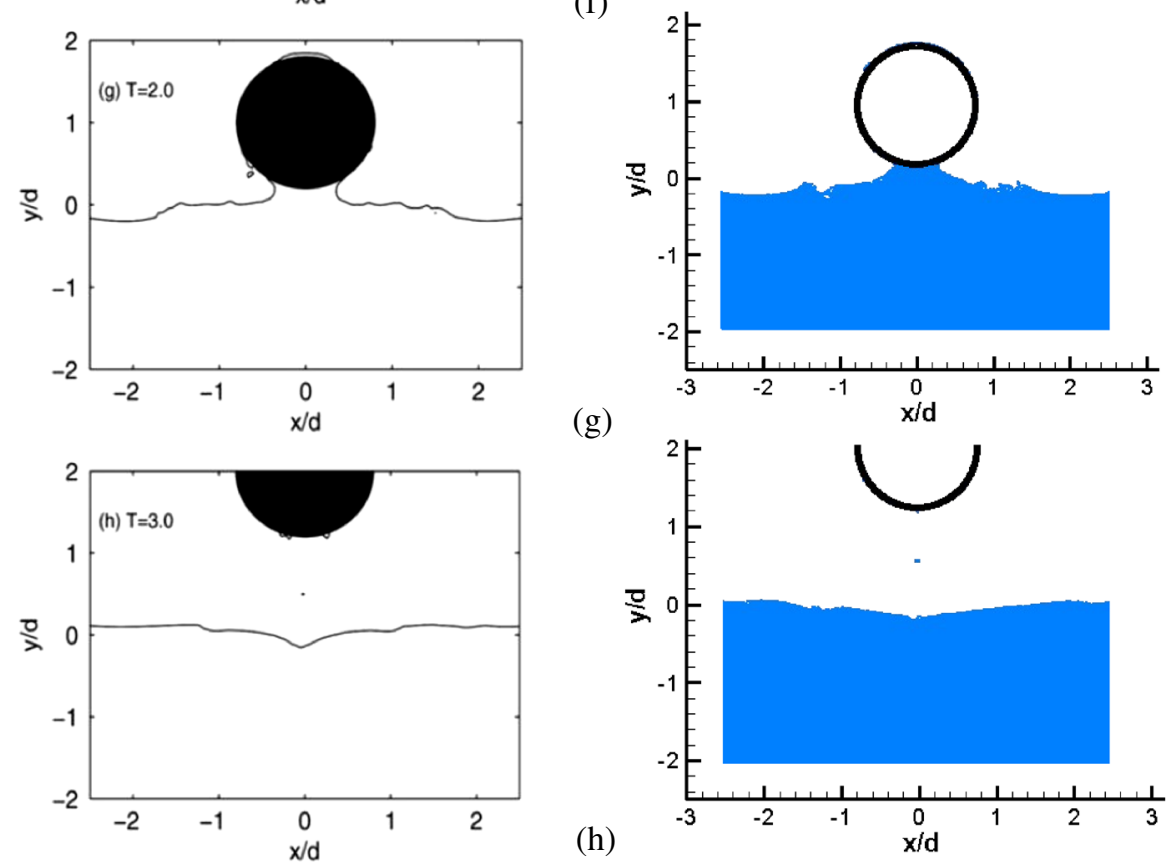

(h)

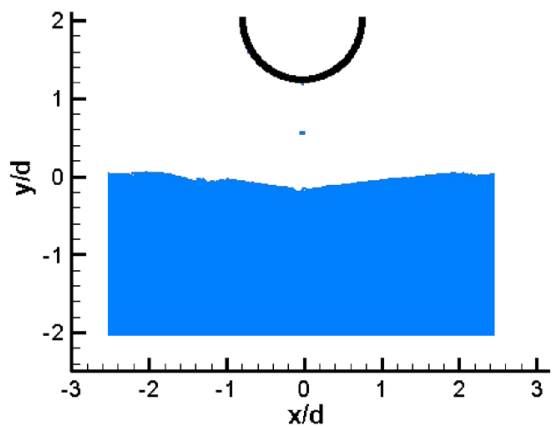

Figure 2. Continued.

Their theory works well in modeling the free surface shape before the cylinder exits the bulk water, while it is not valid when the free surface breaks up.

In our work, the problem setup is the same as those in Greenhow and Moyo's, and Lin's work. In the water exit problem, the radius of the horizontal cylinder is chosen to be $a=1.0 \mathrm{~m}$ and its center at a distance below the still water surface of $d=1.25 \mathrm{~m}$. The gravitational acceleration is fixed to be $g=1.0 \mathrm{~m} / \mathrm{s}^{2}$, and the impulsive upward velocity is applied to the cylinder at $V=0.39 \mathrm{~m} / \mathrm{s}$. Approximately 200,000 particles are used in the SPH simulation with initial particle spacing of $0.025 \mathrm{~m}$ and a time step $\Delta t=0.2 e-3 \mathrm{~s}$. In order to compare the SPH results with existing results $[1,4,36]$ at corresponding time instants, the physical time is non-dimensionalized as $T=|V t / d|$. 
Figure 2 shows the snapshots of the SPH results (right column) and existing numerical and theoretical results (left column) at $T=0.0,0.2,0.4,0.6,0.8,1.0,2.0$ and 3.0. It is clear that SPH results agree well with existing numerical results for the free surface morphology and position of the horizontal cylinder. All numerical results are also close to the theoretical results at earlier stages (before $T=0.4$ ), but difference gradually becomes larger as time marches to 0.6 , after which the theoretical results are no longer valid, and the free surface begins to breakup. When time further increases, and the cylinder moves to higher positions, water begins to detach from around

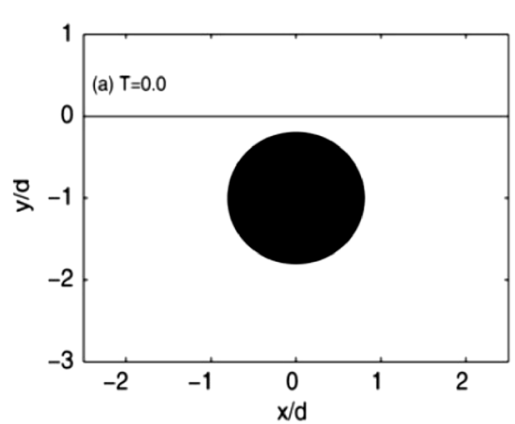

(a)
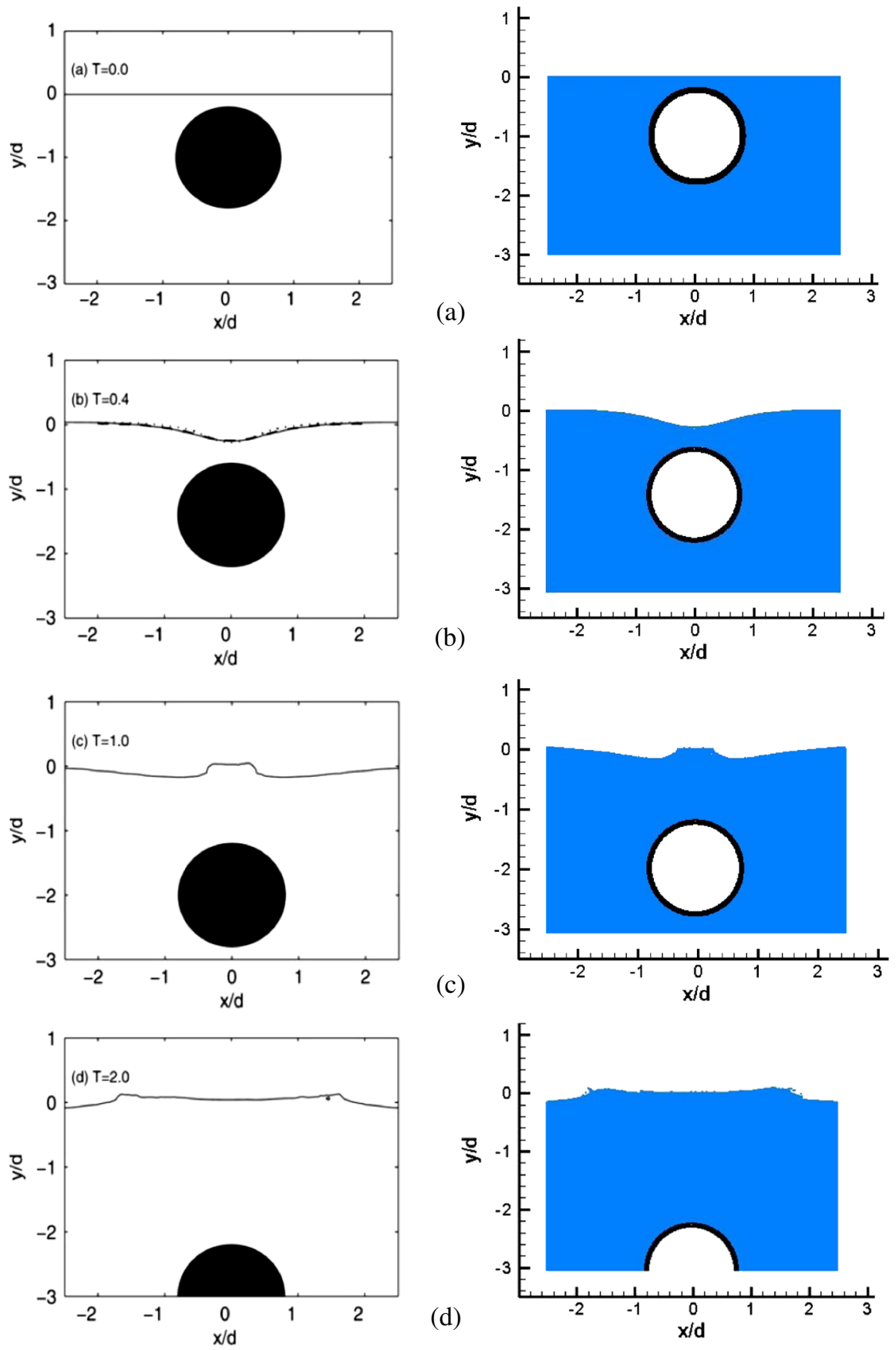

Figure 3. Simulation results of the sinking of a submerged cylinder at different time instants (a: $\mathrm{T}=0.0$; $\mathrm{b}: \mathrm{T}=0.4 ; \mathrm{c}: \mathrm{T}=1.0$ and $\mathrm{d}: \mathrm{T}=2.0$ ). Left: numerical results by Lin (solid line), numerical results by Greenhow and Moyo (dashed line) and theoretical results by Tyvand and Miloh (dotted line) (from [1]). Right: present SPH results. 
the surface of the cylinder, falls down to bulk water and forms water splash with violent free surface deformation $(T=1.0)$. At around $T=2.0$, the cylinder is to exit from the bulk water, water moves to the middle space evacuated by the upward moving cylinder, and this leads to the rise of water level in middle region. It is noted that the SPH method can not only well predict the free surface morphology and position of the horizontal cylinder before its exit from water but also well describe the water detachment, breakup, splash and wave generation during the exit of cylinder from water.

\subsection{Sinking of a submerged cylinder}

The geometry of this example is the same as the previous example while the initially submerged cylinder moves in a downward speed of $V=0.39 \mathrm{~m} / \mathrm{s}$. Figure 3 shows the snapshots of the SPH results (right column) and existing numerical and theoretical results (left column) at $T=0.0,0.4$, 1.0 and 2.0. Again, the SPH results agree well with existing numerical and theoretical results. With the downward movement of the cylinder, water on the top of the cylinder also falls down and forms an apparent valley (at $T=0.4$ ). As time further increases, water from both sides moves to the center of the valley, forms a region with enhanced water level (at $T=1.0)$ and generates water waves to
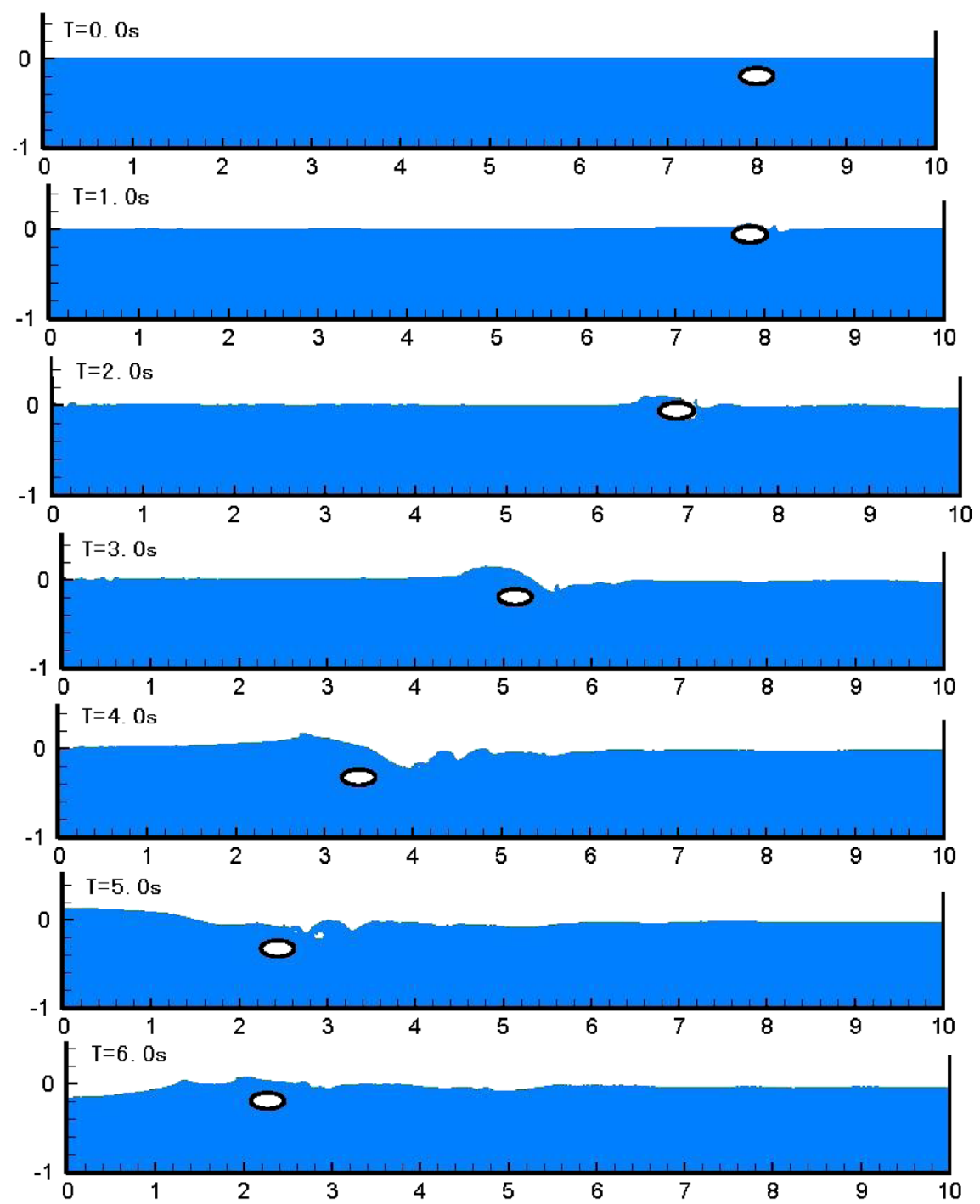

Figure 4. Particle distribution of the movement of an elliptical cylinder near free surface at 0.0, 1.0, 2.0, 3.0, 4.0, 5.0 and $6.0 \mathrm{~s}$. 

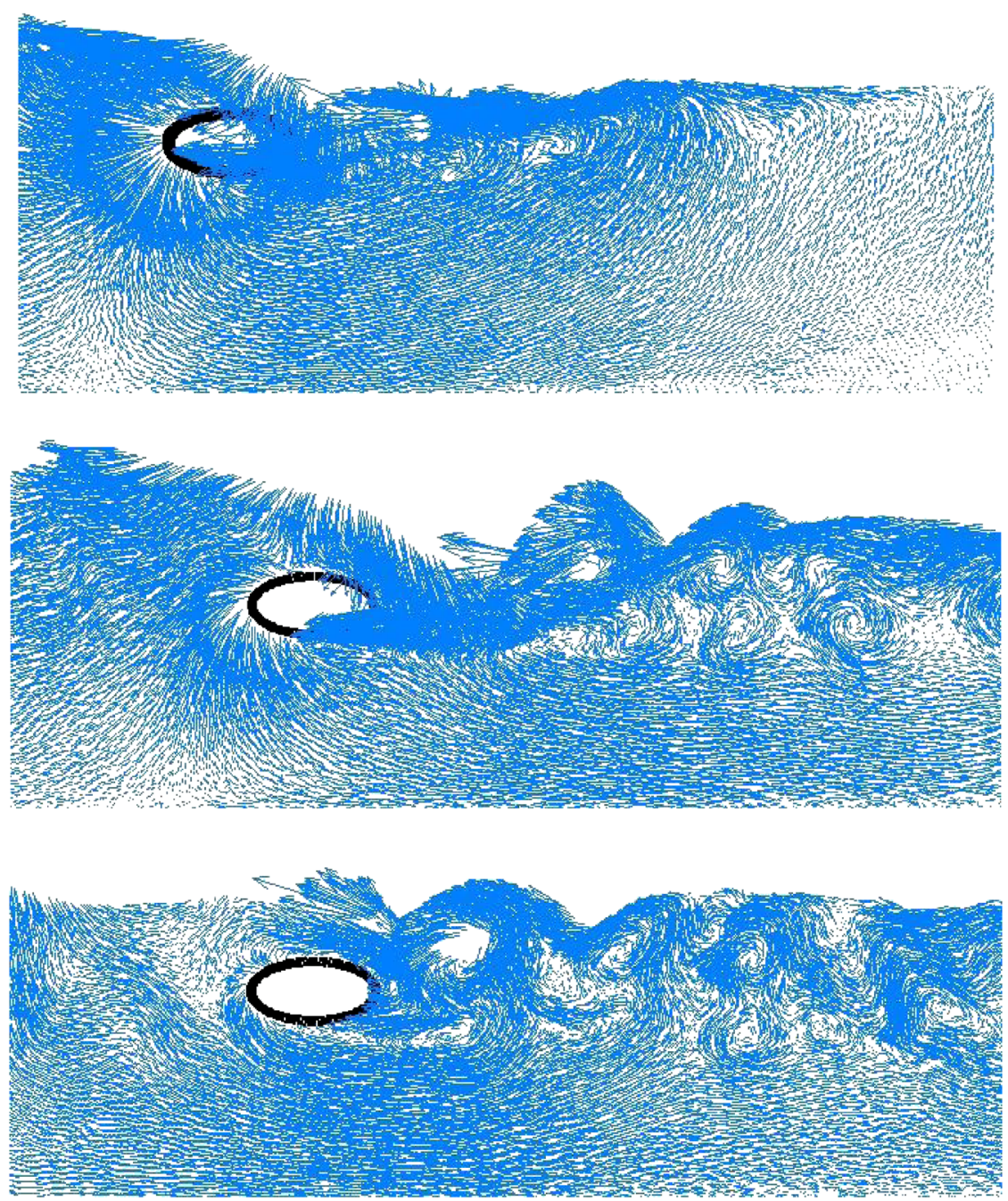

Figure 5. Velocity vectors at $3.0 \mathrm{~s}$ (top), $4.0 \mathrm{~s}$ (middle) and $5.0 \mathrm{~s} \mathrm{(bottom).}$

both sides (at $T=2.0$ ). It is further demonstrated that the present SPH model can well predict the fluid flow interacting with moving rigid body including free surface deformation with breakup and evolution of the cylinder.

\subsection{The movement of an elliptical cylinder near free surface}

In this section, the SPH model will be used to simulate the complicated movement of an elliptical cylinder near the free surface with prescribed acceleration and velocity. Early approaches for investigating waves generated by an accelerating rigid body are generally based on potential flow theory, which is not valid for long-time movement with vortices. Lin provided a numerical simulation of this problem by solving the genetic RANS equation with a cut-cell technique with a fixed grid [1]. The present SPH model also incorporates RANS turbulence model. The effectiveness of the SPH method as well as the RANS turbulence model will be examined.

In the simulation, approximately $100,000 \mathrm{SPH}$ particles are regularly distributed in a computational domain of $10 \mathrm{~m}$ long and $1.0 \mathrm{~m}$ high with initial particle spacing of $0.01 \mathrm{~m}$. The time step is taken as $0.2 \mathrm{e}-3 \mathrm{~s}$. If taking the top-right corner as origin, the elliptical surface of the cylinder can be defined by $(x-8)^{2} / 0.2^{2}+(y+0.2)^{2} / 0.1^{2}=1$ with the center of the cylinder located at $(8,-0.2)$. The elliptical cylinder moves leftwards from stationary with a periodic acceleration and deceleration as $a_{x}=-\sin \omega t$, where $\omega=-2 \pi / T_{p}$ with $T_{p}=6 \mathrm{~s}$. The impulsive horizontal and vertical 


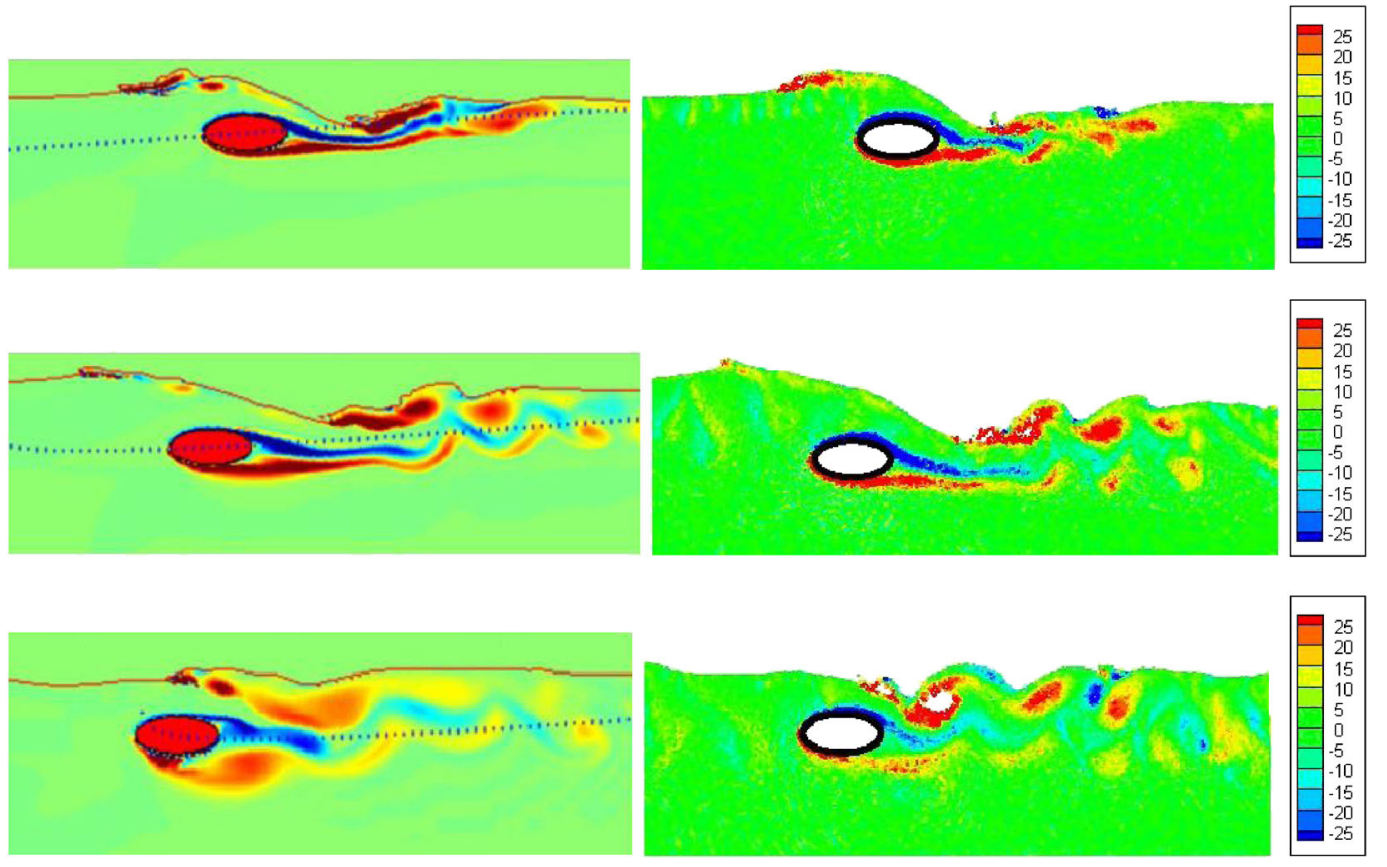

Figure 6. Numerical results (vorticity) of the moving elliptical cylinder at $3.0 \mathrm{~s} \mathrm{(top),} 4.0 \mathrm{~s}$ (middle) and $5.0 \mathrm{~s}$ (bottom). Left: results from [1]. Right: present SPH results.

velocities are therefore $V_{x}=\omega^{-1} \cos w t-w^{-1}$ and $V_{y}=0.16 \cos \omega t$, respectively. According to the periodically changing horizontal and vertical speed, the cylinder will move up for around $0.15 \mathrm{~m}$ and then exit from the free surface and then dives into the water, producing complicated flow pattern with pronounced vortices. The problem setup is the same as that in Lin's work except that the water height is $2.0 \mathrm{~m}$ smaller than that $(3 \mathrm{~m})$ in Lin's work in order to save computational effort.

Figure 4 shows the particle distribution of movement of an elliptical cylinder near free surface at $0.0,1.0,2.0,3.0,4.0,5.0$ and $6.0 \mathrm{~s}$. The evolution of free surface and position of the elliptical cylinder are very close to the numerical results provided in [1]. The cylinder starts to move with gradually increasing speed which reaches maximum at $T=3 \mathrm{~s}$. During this period, waves are generated in front and behind the cylinder because of the interaction of the body motion and fluid flow. The front water wave can overtop the cylinder, impact onto water behind the cylinder and produce violent free surface deformation with water splash. The interaction of the elliptical cylinder with the fluid flows induces vortices with obvious vertex shedding. The long period of movement, together with periodical acceleration and deceleration and elliptical shape, makes the vortices generation and shedding in a complicated manner. After $T>3 \mathrm{~s}$, the cylinder gradually slows down because of the deceleration, and the wave front still keeps moving with a flattened crest. The overtopping and impacting of front water onto behind water will further produce more complicated flow pattern with cavity. Figure 5 shows the zoomed in plots of velocity vector at 3.0, 4.0, 5.0 and $6.0 \mathrm{~s}$. It is clear that a larger number of vortices with different strength are produced. Figure 6 shows the quantitative comparisons of the present SPH results (vorticity) with the numerical results provided in [1]. In general, the vorticity fields obtained from two different approaches are very close. The SPH vorticity field is not smooth in some areas, and this is due to the immature post-processing techniques in meshfree particle methods.

\section{CONCLUSIONS}

This paper presents an SPH model for simulating free surface flows with moving objects. Fluid particles are used to model the free surface flows, and solid particles are used to model the dynamic 
movement of moving rigid objects. The interaction of the neighboring fluid and solid particles renders the fluid-solid interaction and the non-slip solid boundary conditions.

The presented SPH method is improved with density correction and KGC, which leads to better accuracy especially for non-uniformly distributed particles. A coupled dynamic boundary treatment algorithm is used, which include two types of virtual particles to describe the solid boundary, either fixed in computational domain or moving with the rigid object. The kernel-like, soft repulsive force between approaching fluid and solid particles, on one hand, can effectively prevent particles from unphysical penetration, and on the other hand, can avoid unwanted numerical oscillations in solid boundary area. It is also feasible to apply high-order SPH schemes to approximate field functions of virtual solid particles to obtain better accuracy enhance computational accuracy in boundary areas. In order to model flows with strong turbulence and vortices, the RANS turbulence model is incorporated into the SPH method

The model is then applied to simulate three numerical examples including the water exit of a cylinder, the sinking of a submerged cylinder and the complicated motion of an elliptical cylinder near free surface. The present SPH results are comparatively examined with results from other sources. For the first two examples, it is demonstrated that the presented SPH model can well predict the fluid flow interacting with moving rigid body including free surface deformation with breakup and evolution of the cylinder. The last example shows the effectiveness of the presented SPH model in capturing strong turbulence and vortices. It is expected that with multi-phase and multimaterial treatment models, the presented SPH method can be applied to simulate more complicated fluid-structure interaction problems with air entrapment and multiple moving bodies.

\section{ACKNOWLEDGEMENTS}

This work has been supported by the National Natural Science Foundation of China (11172306, 11232012, 81271650, the New Century Excellent Talents in University (NCET-10-0041) and the 100 Talents Program of the Chinese Academy of Sciences.

\section{REFERENCES}

1. Lin PZ. A fixed-grid model for simulation of a moving body in free surface flows. Computers \& Fluids 2007; 36(3):549-561.

2. Anju A, Maruoka A. Kawahara M. 2-D fluid-structure interaction problems by an arbitrary Lagrangian-Eulerian finite element method. International Journal of Computational Fluid Dynamics 1997; 8(1):1-9.

3. Nomura T, Hughes TJR. An arbitrary Lagrangian-Eulerian finite rigid element method for interaction of fluid and a rigid body. Computer Methods in Applied Mechanics and Engineering 1992; 95(1):115-138.

4. Greenhow M, Moyo S. Water entry and exit of horizontal circular cylinders. Philosophical Transactions of the Royal Society of London. Series A: Mathematical, Physical and Engineering Sciences 1997; 355(1724):551-563.

5. Lucy LB. A numerical approach to the testing of the fission hypothesis. Astronomical Journal 1977; 82(12):1013-1024.

6. Gingold RA, Monaghan JJ. Smoothed particle hydrodynamics-theory and application to non-spherical stars. Monthly Notices of the Royal Astronomical Society 1977; 181:375-389.

7. Liu GR, Liu MB. Smoothed Particle Hydrodynamics: A Meshfree Particle Method. World Scientific: Singapore, 2003.

8. Liu MB, Liu GR, Lam KY. Constructing smoothing functions in smoothed particle hydrodynamics with applications. Journal of Computational and Applied Mathematics 2003; 155(2):263-284.

9. Oger G, Doring M, Alessandrini B, Ferrant P. Two-dimensional SPH simulations of wedge water entries. Journal of Computational Physics 2006; 213(2):803-822.

10. Shao SD. Incompressible SPH simulation of water entry of a free-falling object. International Journal for Numerical Methods in Fluids 2009; 59(1):91-115.

11. Iglesias AS, Rojas LP, Rodriguez RZ. Simulation of anti-roll tanks and sloshing type problems with smoothed particle hydrodynamics. Ocean Engineering 2004; 31(8-9):1169-1192.

12. Rhee SH, Engineer L. Unstructured grid based Reynolds-averaged Navier-Stokes method for liquid tank sloshing. Journal of Fluids Engineering 2005; 127:572.

13. Marrone S, Colagrossi A, Antuono M, Lugni C, Tulin MP. A 2D+t SPH model to study the breaking wave pattern generated by fast ships. Journal of Fluids and Structures 2011; 27(8):1199-1215.

14. Catalano P, Amato M. An evaluation of RANS turbulence modelling for aerodynamic applications. Aerospace Science and Technology 2003; 7(7):493-509.

15. Anderson J, Wendt J. Computational Fluid Dynamics. McGraw-Hill: New York, 1995. 
16. Monaghan JJ. Smoothed particle hydrodynamics. Reports on Progress in Physics 2005; 68(8):1703-1759.

17. Liu MB, Liu GR. Smoothed particle hydrodynamics (SPH): an overview and recent developments. Archives of Computational Methods in Engineering 2010; 17(1):25-76.

18. Chen Z, Zong Z, Liu MB, Li HT. A comparative study of truly incompressible and weakly compressible SPH methods for free surface incompressible flows. International Journal for Numerical Methods in Fluids 2013; 73(9):813-829.

19. Shao SD, Ji CM, Graham DI, Reeve DE, James PW, Chadwick AJ. Simulation of wave overtopping by an incompressible SPH model. Coastal Engineering 2006; 53(9):723-735.

20. Cummins SJ, Rudman M. An SPH projection method. Journal of Computational Physics 1999; 152:584-607.

21. Rafiee A, Thiagarajan KP. An SPH projection method for simulating fluid-hypoelastic structure interaction. Computer Methods in Applied Mechanics and Engineering 2009; 198:2785-2795.

22. Liu X, Xu HH, Shao SD. Lin PZ. An improved incompressible SPH model for simulation of wave-structure interaction. Computers \& Fluids 2013; 71:113-123.

23. Chen JK, Beraun JE. A generalized smoothed particle hydrodynamics method for nonlinear dynamic problems. Computer Methods in Applied Mechanics and Engineering 2000; 190(1-2):225-239.

24. Liu MB, Liu GR. Restoring particle consistency in smoothed particle hydrodynamics. Applied Numerical Mathematics 2006; 56(1):19-36.

25. Liu MB, Xie WP, Liu GR. Modeling incompressible flows using a finite particle method. Applied Mathematical Modelling 2005; 29(12):1252-1270.

26. Colagrossi A, Landrini M. Numerical simulation of interfacial flows by smoothed particle hydrodynamics. Journal of Computational Physics 2003; 191(2):448-475.

27. Dilts GA. Moving-least-squares-particle hydrodynamics-i. consistency and stability. International Journal for Numerical Methods in Engineering 1999; 44(8):1115-1155.

28. Dilts GA. Moving-least-squares-particle hydrodynamics-ii. conservation and boundaries. International Journal for Numerical Methods in Engineering 2000; 48(10):1503-1524.

29. Liu MB, Shao JR, Chang JZ. On the treatment of solid boundary in smoothed particle hydrodynamics. Science China Technological Sciences 2012; 55(1):244-254.

30. Zheng X, Duan WY, Ma QW. A new scheme for identifying free surface particles in improved SPH. Science China Physics, Mechanics and Astronomy 2012; 55(8):1454-1463.

31. Monaghan JJ. Simulating free surface flows with SPH. Journal of Computational Physics 1994; 110:399-399.

32. Morris J, Fox P, Zhu Y. Modeling low Reynolds number incompressible flows using SPH. Journal of Computational Physics 1997; 136(1):214-226.

33. Shao JR, Li HQ, Liu GR, Liu MB. An improved SPH method for modeling liquid sloshing dynamics. Computers \& Structures 2012; 100-101:18-26.

34. Koumoutsakos P. Multiscale flow simulations using particles. Annual Review of Fluid Mechanics 2005; 37:457-487.

35. Liu MB, Shao JR, Li HQ. Numerical simulation of hydro-elastic problems with smoothed particle hydrodynamics. Journal of Hydrodynamics 2013; 25(5):840-847.

36. Tyvand PA, Miloh T. Free-surface flow due to impulsive motion of a submerged circular cylinder. Journal of Fluid Mechanics 1995; 286(1):67-101. 